\title{
Announcement of awards by the Journal of Plant Research
}

\author{
Hirokazu Tsukaya
}

Published online: 26 July 2011

(C) The Botanical Society of Japan and Springer 2011

I am very pleased to announce that the two following papers won the Journal of Plant Research (JPR) Best Paper Award 2011:

1. Hiroyuki Muraoka, Nobuko Saigusa, Kenlo N. Nasahara, Hibiki Noda, Jun Yoshino, Taku M. Saitoh, Shin Nagai, Shohei Murayama, and Hiroshi Koizumi (2010) Effects of seasonal and interannual variations in leaf photosynthesis and canopy leaf area index on gross primary production of a cool-temperate deciduous broadleaf forest in Takayama, Japan. J Plant Res 123(4):563-576

2. Hidenori Tsuboi and Masamitsu Wada (2010) Speed of signal transfer in the chloroplast accumulation response. J Plant Res 123 (3):381-390.

These award-winning works were selected from all the original papers published in the Journal of Plant Research in 2010 by votes of the editorial board members according to the JPR Awards Selection Procedure. Here I would like to briefly introduce the contents of the two papers.

In Muraoka et al. (2010), the authors investigated the seasonal dynamics of carbon dioxide exchange between the atmosphere and deciduous forests, examining the effects of temporal variations of the canopy leaf area index (LAI) and leaf photosynthetic capacity on gross primary production from data on a cool-temperate deciduous broadleaf forest in Japan. They carried out simulation analysis and showed that the phenology of leaf photosynthetic capacity and LAI must be considered to analyze and predict carbon fixation

H. Tsukaya ( $\square)$

Graduate School of Science, The University of Tokyo,

Science Building \#2, 7-3-1 Hongo, Bunkyo-ku,

Tokyo 113-0033, Japan

e-mail: jpreic@biol.s.u-tokyo.ac.jp in forest ecosystems. Because carbon dynamics is one of the most important issues in the world now, this study will provide an important basis for future studies in the field.

In Tsuboi and Wada (2010), the authors analyzed the speeds of signal transfer from light perceptions to chloroplast movements by time-lapse imaging and a microbeam irradiation system. They discovered that movement speeds under red or blue light were similar; however, speeds varied according to cell polarity in protonemal cells in Adiantum capillus-veneris. Interestingly, they found that chloroplasts located farthest from the microbeam were found to move faster than those close to the site of the irradiation. The phenomenon was found to be common between Adiantum capillus-veneris, a fern, and Arabidopsis thaliana, an angiosperm. This study is a good representative of Japanese photobiology, which has a long history.

In addition to the above-mentioned awards, the winner of the Most-Cited Paper Award is selected from all the papers published in 2008 by reference to the ISI database. That award was won by the following paper:

- Toru Tokuoka (2008) Molecular phylogenetic analysis of Violaceae (Malpighiales) based on plastid and nuclear DNA sequences. J Plant Res 121 (3):253-260.

In this paper, the author presented an analysis of the molecular phylogeny of Violaceae using sequences from rbcL, atpB, matK, and 18SrDNA from 19 genera. This phylogenetic analysis with high resolution revealed evolutionary trends of flower morphology, seed character, and other morphological characters in this family. The study makes a significant impact on understanding the evolution not only of this family but also of angiosperms, and was cited 14 times in 2009 and 2010 according to the ISI database. Considering the smaller number of citations in 
this research field than in other fields, the relatively high citation figure itself is evidence of the impact of this study. Thanks to such good papers published in JPR, the latest impact factor (year 2010) of the JPR was 1.512.

As exemplified by the above-mentioned three articles, JPR has published outstanding papers from a broad spectrum of research fields, papers that are cited by many plant scientists. Indeed, reflecting the broad scope of our journal, which covers a wide range of plant sciences from taxonomy, anatomy, physiology, and ecology, to molecular genetics, the two Best Paper awardees are in the fields of ecophysiology and photobiology, respectively, and the Most-Cited Paper awardee is in the field of evolutionary biology.
This year we published a special issue on abscisic acid (ABA), inviting articles on studies of ABA. Members of the Botanical Society of Japan, which publishes JPR, have contributed much to recent important advances in understanding phytohormones as represented by ABA, and I hope this will be a key basis for future studies in the field. By continuing to publish excellent papers and reviews with great impact, JPR makes an ongoing contribution to the plant science field.

Hirokazu Tsukaya Editor-in-Chief, Journal of Plant Research 